\title{
Transport in Bilayer Graphene near Charge Neutrality: Which Scattering Mechanisms Are Important?
}

\author{
Glenn Wagner 0 , Dung X. Nguyen, and Steven H. Simon \\ Rudolf Peierls Centre for Theoretical Physics, Parks Road, Oxford OX1 3PU, United Kingdom
}

(Received 7 June 2019; published 13 January 2020)

\begin{abstract}
Using the semiclassical quantum Boltzmann equation (QBE), we numerically calculate the dc transport properties of bilayer graphene near charge neutrality. We find, in contrast to prior discussions, that phonon scattering is crucial even at temperatures below $40 \mathrm{~K}$. Nonetheless, electron-electron scattering still dominates over phonon collisions allowing a hydrodynamic approach. We introduce a simple two-fluid hydrodynamic model of electrons and holes interacting via Coulomb drag and compare our results to the full QBE calculation. We show that the two-fluid model produces quantitatively accurate results for conductivity, thermopower, and thermal conductivity.
\end{abstract}

DOI: 10.1103/PhysRevLett.124.026601

Graphene has attracted an enormous amount of attention in the last decade [1]. While its transport properties have been extensively studied [2], other related materials are only beginning to be examined. Advances in nanotechnology have recently allowed electrical measurements on suspended bilayer graphene (BLG) samples [3-11]. Motivated by these advances, we theoretically examine the dc transport properties of BLG using a quantum Boltzmann equation (QBE) approach. Solving the QBE is a numerically daunting task. We show that in many cases a far simpler two-fluid model, which we introduce in this Letter, accurately describes the QBE results.

Measurement of the electrical conductivity in BLG has been reported in Ref. [3] and we show that our QBE results agree with the experimental data over a wide range of parameters. We focus our calculation on the regime explored in that experiment, i.e., close to charge neutrality and at temperatures $T \sim 10 \mathrm{~K}-40 \mathrm{~K}$ (using the common Bernal stacked BLG with no out of plane field). We show that in this regime, surprisingly, the effect of phonon scattering plays an essential role (with the effect of finite sample size playing a minor role). We then use the QBE to make predictions for the thermopower and thermal conductivity that can be verified in future experiments. As in the case of monolayer graphene [12,13], we expect that electrons in BLG will have high mobility and scattering of electrons among themselves will be the dominant scattering mechanism. We thus expect that the QBE will reduce to some form of hydrodynamics [14-20]. Indeed we show that our transport results can be quantitatively understood with an extremely simple two-fluid hydrodynamic model [21-24]. This simplification allows intuitive understanding of the physics that is not otherwise possible.

In this Letter we begin by briefly discussing our QBE method, which we elaborate in detail in a companion paper [25]. This approach is similar to previous work on the conductivity of monolayer graphene [26,27], two coupled monolayers [28], and BLG [29,30]. Compared to Ref. [29], which studies the case of clean BLG, we calculate the conductivity away from charge neutrality $(\mathrm{CN})$, which requires including additional scattering mechanisms. The paper [30] does study the conductivity of BLG away from $\mathrm{CN}$ by including the effect of disorder; however we aim to provide a more quantitative analysis allowing for comparison with experimental data. We compare our results for the electrical conductivity to the experimental results from Ref. [3] in order to extract the value of the only free parameter of the theory: the phonon scattering rate. The value we determine for the phonon deformation potential lies within the range reported by previous authors. We then use this value to calculate the thermal conductivity and the thermoelectric coefficient. Finally, we introduce the twofluid model and show that it agrees quantitatively with the more detailed QBE numerics.

$Q B E$.-At low energies, bilayer graphene can be described in a two-band model with a quadratic dispersion $\epsilon_{\lambda}(k)=\lambda|\mathbf{k}|^{2} / 2 m$ where $\lambda=+(-)$ for electrons in the conduction (valence) band [25,31], $m=0.033 m_{e}$ is the effective mass [32], and $\mathbf{k}$ is the two-dimensional momentum. There are two valleys $\left(K\right.$ and $\left.K^{\prime}\right)$ and two spin states in each valley, thus giving $N_{f}=4$ flavors. We neglect additional effects at low temperatures such as trigonal warping [33] and opening of an interaction induced gap [34], restricting the applicability of our approach to $T \gtrsim 10 \mathrm{~K}$ [3].

The QBE is a semiclassical approach which is valid when the de Broglie wavelength is much shorter than the scattering lengths. This is equivalent to the condition $\alpha_{x} \equiv \beta \tau_{x}^{-1} \ll 1$, where $\tau_{x}$ stands for the scattering time due to any of the scattering mechanisms in the problem, and $\beta$ is the inverse temperature. 
In equilibrium the occupancy of a $\mathbf{k}$ state is given by the usual Fermi factor. The QBE determines the change in occupancy of the $\mathbf{k}$ states due to small perturbations such as an external electric field $\mathbf{E}$ or temperature gradient $\nabla T$. Formally the QBE is derived from the Kadanoff-Baym equations for the evolution of Green's functions using a Born collision integral to describe scattering. Once Boltzmann equations are formally derived, the solution is obtained by expanding the $\mathbf{k}$ space occupation function $f(\mathbf{k})$ in a set of basis functions, which then reduces the QBE to a set of linear equations which can be numerically solved. The size of the basis set is then expanded to convergence [27-29,35]. This method of solution for the Boltzmann equation is well known in the plasma physics community and goes by the name Spitzer-Härm method [36]. Details of both the derivation of the QBE, and the method of solution are presented in detail in Ref. [25] and the Supplemental Material [37].

We calculate the electrical current $\mathbf{J}$ and the heat current, which is defined as $\mathbf{Q}=\mathbf{J}^{E}-(\mu / e) \mathbf{J}$, where $\mathbf{J}^{E}$ is the energy current and $e<0$ is the electron charge. From this we can determine the electrical conductivity $\sigma$, the thermal conductivity $K$ and the thermoelectric coefficient $\Theta$ by

$$
\left(\begin{array}{l}
\mathbf{J} \\
\mathbf{Q}
\end{array}\right)=\left(\begin{array}{cc}
\sigma & \Theta \\
T \Theta & K
\end{array}\right)\left(\begin{array}{c}
\mathbf{E} \\
-\nabla T
\end{array}\right) .
$$

The open circuit thermal conductivity [38] which is usually measured in experiments is given by $\kappa=K-T \Theta \sigma^{-1} \Theta$.

Coulomb and phonon scattering.-We first consider Coulomb (electron-electron, electron-hole) scattering, which we expect to be dominant. We use the form of the screened Coulomb potential valid in the experimentally relevant regime $\beta \mu \lesssim 1$. In the experimental data [3], to a good approximation the conductivity only depends on the dimensionless combination $\beta \mu$. (see Fig. 1 [39]). If only Coulomb interactions are included then $\beta \mu$ is the only dimensionless parameter of the problem (the electromagnetic fine-structure constant cancels out when the screened potential is used [37]). However, away from $\mathrm{CN}$, the socalled momentum mode (i.e., a simple Galilean boost), where electrons and holes move in the same direction, carries electrical current, and is not relaxed by Coulomb scattering since it conserves momentum. Thus, to obtain a finite conductivity, another scattering mechanism that relaxes momentum must be considered. We identify three possible such mechanisms: impurity scattering, scattering off the boundary of the finite-size sample, and phonons. In the former two cases, the relevant dimensionless scattering parameter $\alpha_{x}$ depends on temperature [40]. Therefore, if either of these scattering mechanisms were most important (after Coulomb scattering) the curves for different temperatures would not collapse when plotted as a function of $\beta \mu$, and this does not agree with the experimental data. We thus disregard these two scattering mechanisms. The situation,

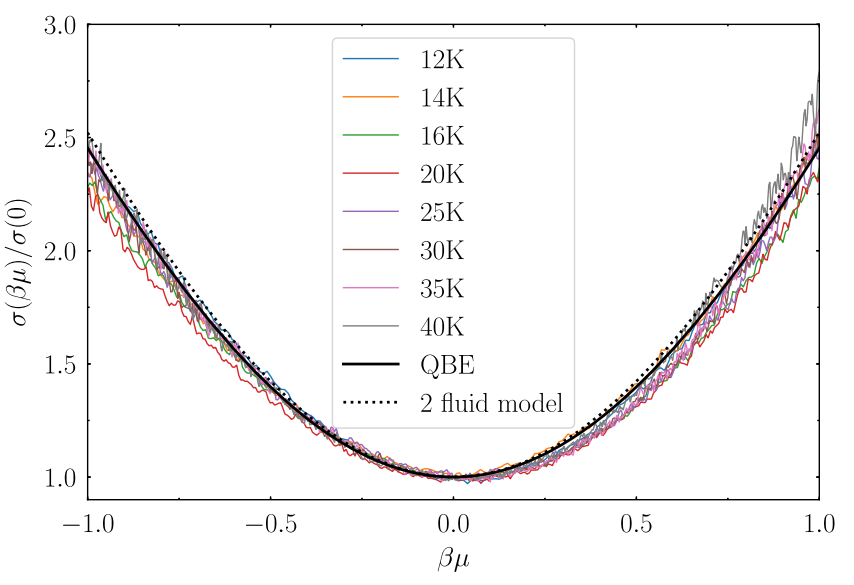

FIG. 1. Electrical conductivity $\sigma(\beta \mu)$ for different values of the temperature. From the experimental data, we fit the value $\alpha_{\mathrm{ph}}=0.05$. The experimental data are from Ref. [3]. The black solid curve is the result of the QBE calculation. The black dashed curve shows the result from the two-fluid model and shows good agreement with the full QBE calculation.

however, is different for phonon scattering. The experiment is at temperatures above the Bloch-Grüneisen temperature and hence the longitudinal acoustic phonons have a scattering time $[41,42]$

$$
\tau_{\mathrm{ph}}^{-1}=\frac{D^{2} m k_{B} T}{2 \rho c^{2}}
$$

where $c$ is the speed of sound in graphene, $D$ is the deformation potential, and $\rho$ is the mass density of BLG. The relevant dimensionless parameter $\alpha_{\mathrm{ph}}=\beta \tau_{\mathrm{ph}}^{-1}$ is then independent of temperature and should result in the conductivity being a function of $\beta \mu$ only, in close agreement with experiment [3]. We emphasize the surprising result that even at these comparatively low temperatures of $12-40 \mathrm{~K}$, phonons provide the primary momentum relaxation mechanism.

Electrical conductivity.-In the absence of a magnetic field the conductivity $\sigma_{i j}=\left(N_{f} e^{2} / 2 \hbar\right) \tilde{\sigma} \delta_{i j}$ depends on the dimensionless numbers $\beta \mu$ and $\alpha_{\mathrm{ph}}$. The thermal density of electrons (holes) for the free Fermi gas is given by $n^{e(h)}=$ $\left[N_{f} m /(2 \pi \beta)\right] \ln \left(1+e^{ \pm \beta \mu}\right)$ and as we increase $|\beta \mu|$ there are more charge carriers. However the total number density of electrons plus holes only increases by $17 \%$ between $|\beta \mu|=0$ and 1 (at fixed $T$ ), and therefore this effect alone is too small to account for the electrical conductivity more than doubling in this range. To understand this we realize that the primary scattering is electron-hole collisions [3] and therefore the conductivity at large chemical potential is large both because we have many electrons to carry the charge and also because there are not many holes to scatter from. A nonzero $\alpha_{\mathrm{ph}}$ makes the conductivity finite and, as mentioned above, the curves for different temperatures 
approximately collapse when plotted as a function of $\beta \mu$. We treat the deformation potential $D$ as a fit parameter being that various different approaches have given different estimates of this quantity [43-47]. Our best fit value $\alpha_{\mathrm{ph}}=$ 0.05 corresponds to $D \approx 27 \mathrm{eV}$, which is consistent with prior expectation that it lies in the range $10-30 \mathrm{eV}$. Including the effect of finite size (boundary scattering) reduces the phonon scattering required to match the experiment. Taking account of the fact that the typical size of the system is around $3 \mu \mathrm{m}$, and there will be additional momentum-relaxing scattering off the boundary of the sample, the best fit $D$ may be reduced by around $30 \%$.

In Fig. 1 we show $\sigma$ as a function of $\beta \mu$. We show both the result of QBE calculation using the above discussed fit value of $D$ as well as the experimental data from Ref. [3].

Exactly at $\mathrm{CN}$, we can compare our prediction for the magnitude of $\sigma$ to prior calculations by Ref. [29]. Our work differs in three respects from Ref. [29]. First, they take the screening wave vector $q_{T F}$ to be constant, second they do not make the approximation $q_{T F} \gg q$ in the screened potential, and third they have not included all the necessary matrix elements for BLG. In order to compare our work, we ignore the momentum-relaxing scattering mechanisms since Ref. [29] does not include those and the conductivity is well defined without them at CN. Reference [29] finds $\sigma^{\mathrm{CN}}=\left(e^{2} / h\right)(27.4+0.353 \sqrt{T[\mathrm{~K}]})$, which has a large constant piece and a smaller piece that depends on temperature. On the other hand, from dimensional analysis, in a clean system our result at $\mathrm{CN}$ does not depend on temperature. This discrepancy arises from making the approximation $q_{T F} \gg q$. However, in an experimental setting, the contributions arising from momentum-relaxing scattering will be the most significant for the temperature dependence, justifying our approximation.

Comparison of our results for the magnitude of $\sigma$ at $\mathrm{CN}$ to experiment is more difficult because different samples give different precise values of conductivity-differing from each other by factors of up to about 4 . Our prediction lies acceptably in the middle of the experimental range.

We can also compare our results to those in Ref. [30], which analyzes the QBE for BLG. We note that Ref. [30] includes only the direct terms in the collision integral; however the effect of the exchange terms is small, justifying the approach of the former paper. A further difference between our work and Ref. [30] is that we use the full momentum-dependent screening wave vector. Overall, we find qualitative agreement with that work.

Thermal properties. - Including only Coulomb scattering, the thermal conductivity $K$ diverges since the momentum mode carries thermal current and cannot be relaxed by Coulomb interactions. Phonons again regulate this divergence. One might expect that the thermal conductivity increases with increasing $|\beta \mu|$ since the total heat transport carrier density $n^{e}+n^{h}$ increases. However, we note that $K$ actually decreases with increasing $|\beta \mu|$ which is
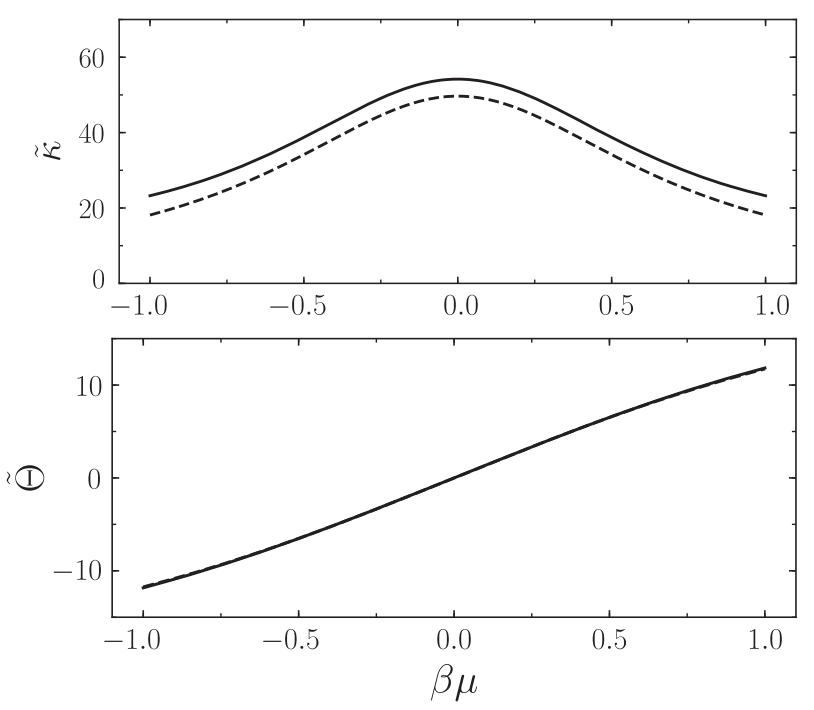

FIG. 2. Top: The dimensionless open-circuit thermal conductivity $\tilde{\kappa}=\left(N_{f} k_{B}^{2} T / 2 \hbar\right)^{-1} \kappa$ calculated using the QBE (solid line) and two-fluid approximation (dashed line). Bottom: The dimensionless thermoelectric coefficient is defined as $\tilde{\Theta}=$ $\left(N_{f} e k_{B} T / 2 \hbar\right)^{-1} \Theta$ calculated using the QBE (solid line) and two-fluid approximation (dashed line). The two curves show near perfect overlap. Both figures use $\alpha_{\mathrm{ph}}=0.05$.

counterintuitive. In the limit of weak phonon scattering we will see below that $K \sim 1 /\left(n^{e}+n^{h}\right)$. The open circuit thermal conductivity $\kappa_{i j}=\left(N_{f} k_{B}^{2} T / 2 \hbar\right) \tilde{\kappa} \delta_{i j}$, plotted in Fig. 2 (top), decreases faster than $K$ away from $\mathrm{CN}$ since the momentum mode carries electric current and hence does not contribute to $\kappa$. The thermoelectric coefficient $\Theta_{i j}=\left(N_{f} e k_{B} T / 2 \hbar\right) \tilde{\Theta} \delta_{i j}$ plotted in Fig. 2 (bottom) vanishes at $\mathrm{CN}$ and increases as we increase $\beta \mu$, as the momentum mode now carries both heat and charge.

Two-fluid model.-Since Coulomb scattering is dominant we expect a hydrodynamic description will be appropriate. Further, since the scattering between electrons and holes is suppressed due to both matrix element effects and energy-momentum conservation constraints [25], we believe treating the electron fluid and the hole fluids as weakly interacting with each other will be accurate. In this limit, due to the strong scattering within each species, each of the two fluids should have a well-defined temperature, chemical potential, and velocity. We thus introduce a twofluid model [21-24], which shows excellent agreement with our detailed numerical calculation [48]. The evolution of the mean fluid velocities of electrons (holes) $\mathbf{u}^{e}\left(\mathbf{u}^{h}\right)$ can then be derived explicitly from the QBE (see Supplemental Material [37] for detailed derivation) giving [49]

$$
\begin{aligned}
& m \dot{\mathbf{u}}^{e}=-\frac{m}{\tau_{e h}}\left(\mathbf{u}^{e}-\mathbf{u}^{h}\right)-\frac{m}{\tau_{\mathrm{ph}}} \mathbf{u}^{e}+e \mathbf{E}-\Lambda^{e} k_{B} \nabla T \\
& m \dot{\mathbf{u}}^{h}=-\frac{m}{\tau_{h e}}\left(\mathbf{u}^{h}-\mathbf{u}^{e}\right)-\frac{m}{\tau_{\mathrm{ph}}} \mathbf{u}^{h}-e \mathbf{E}-\Lambda^{h} k_{B} \nabla T .
\end{aligned}
$$


Here $\tau_{e h}=\tau_{0}\left(n^{e}+n^{h}\right) / n^{h}$ is the scattering time for electrons to scatter off holes and $n^{e} \tau_{e h}=n^{h} \tau_{h e}$ guarantees momentum conservation. By evaluating the Coulomb collision integral we extract the value $\beta \tau_{0}^{-1}=0.15$ (see Supplemental Material [37]). Here, $\Lambda^{e}\left(\Lambda^{h}\right)$ is the entropy per electron (hole) of the free Fermi gas characterized by $\beta \mu$. An explicit expression for these quantities is given in the Supplemental Material [37].

We are interested in dc transport so we may set the left of these two equations to zero, and solve for the velocities $\mathbf{u}^{e, h}$. The electric current is then $\mathbf{J}=e\left(n^{e} \mathbf{u}^{e}-n^{h} \mathbf{u}^{h}\right)$ and the thermal current is $\mathbf{J}^{E}=k_{B} T\left(\Lambda^{e} n^{e} \mathbf{u}^{e}+\Lambda^{h} n^{h} \mathbf{u}^{h}\right)$. The advantage of the two-fluid model is its simplicity which allows us to obtain analytic expressions (see Supplemental Material [37]) for transport coefficients. In the limit $\tau_{\mathrm{ph}} \gg \tau_{0}$ the expressions simplify even more giving, for example, $\tilde{K}=\left(2 \pi^{2} m k_{B} T / 9\right) \alpha_{\mathrm{ph}}^{-1} /\left(n^{e}+n^{h}\right)$. In Figs. 1 and 2 we compare the results from the QBE and the two-fluid model. For the electrical conductivity and the thermopower the agreement is extremely good, whereas for the thermal conductivity we note a slight quantitative disagreement. This can be traced back to the fact that the two-fluid model only includes the first momentum moment of the QBE. In order to account for the thermal current more accurately, the second momentum moment would have to be included as well. Although we only compare the two-fluid model and the QBE results for the experimentally relevant value of $\alpha_{\mathrm{ph}}$, we show in the companion paper [25] that the agreement continues to hold well for a range of $\alpha_{\mathrm{ph}}$.

Since heat is carried by the momentum mode, the Coulomb drag between electrons and holes will not affect it, and the thermal conductivity will mainly be limited by the phonon scattering. The electrical conductivity, on the other hand, will be limited by both phonon scattering and the Coulomb drag between electrons and holes. Therefore, the electrical conductivity is suppressed compared to the thermal conductivity, leading to a large Lorenz number $\mathcal{L} \equiv \kappa /(\sigma T)$. At charge neutrality we find $\mathcal{L} \approx 25\left(k_{B} / e\right)^{2}$ which is much larger than the value of $\pi^{2} / 3\left(k_{B} / e\right)^{2}$ predicted by the Wiedemann-Franz law. The violation of the Wiedemann-Franz law for BLG has been previously pointed out by Ref. [30] and has been experimentally observed for monolayer graphene in Ref. [50].

Conclusion.-In this Letter we have calculated the transport properties of bilayer graphene. Our results for the electrical conductivity match the experimental results in Ref. [3]. From the experimental data we deduce that even at low temperatures, the scattering off phonons is crucial. Nonetheless, the dominant scattering mechanism is between charge carriers of the same species, which justifies a two fluid approach which shows excellent agreement with the detailed numerical results of the QBE and provides a simple way of calculating the transport properties analytically. One can adapt our two fluid model to different experimental setups with slight modifications [25].

It would be interesting to test the predictions for the thermopower and thermal conductivity in upcoming experiments. Our formalism can be expanded to address a variety of other quantities of interest, including finite frequency effects, spin transport, and Hall viscosity.

Statement of compliance with EPSRC policy framework on research data: This publication is theoretical work that does not require supporting research data.

We would like to thank the authors of Ref. [3], YoungWoo Nam, Dong-Keun Ki, David Soler Delgado, and Alberto Morpurgo, for kindly providing the data from that work to us. We are very grateful to David Soler Delgado and Alberto Morpurgo for discussing their experiment with us. We would also like to acknowledge helpful discussions with Philipp Dumitrescu and Lars Fritz. This work was supported by EPSRC Grants No. EP/N01930X/1 and No. EP/S020527/1.

[1] K. S. Novoselov, A. Mishchenko, A. Carvalho, and A. H. Castro Neto, Science 353, aac9439 (2016).

[2] N. Peres, Rev. Mod. Phys. 82, 2673 (2010).

[3] Y. Nam, D.-K. Ki, D. Soler-Delgado, and A. F. Morpurgo, Nat. Phys. 13, 1207 (2017).

[4] D.-K. Ki, V. I. Fal'ko, D. A. Abanin, and A. F. Morpurgo, Nano Lett. 14, 2135 (2014).

[5] R. T. Weitz, M. T. Allen, B. E. Feldman, J. Martin, and A. Yacoby, Science 330, 812 (2010).

[6] B. E. Feldman, J. Martin, and A. Yacoby, Nat. Phys. 5, 889 (2009).

[7] M. T. Pettes, I. Jo, Z. Yao, and L. Shi, Nano Lett. 11, 1195 (2011).

[8] D.-K. Ki and A. F. Morpurgo, Nano Lett. 13, 5165 (2013).

[9] F. Freitag, J. Trbovic, M. Weiss, and C. Schönenberger, Phys. Rev. Lett. 108, 076602 (2012).

[10] J. Velasco, Jr., L. Jing, W. Bao, Y. Lee, P. Kratz, V. Aji, M. Bockrath, C. N. Lau, C. Varma, R. Stillwell, D. Smirnov, F. Zhang, J. Jung, and A. H. MacDonald, Nat. Nanotechnol. 7, 156 (2012).

[11] Y. Nam, D.-K. Ki, M. Koshino, E. McCann, and A. F. Morpurgo, 2D Mater. 3, 045014 (2016).

[12] K. Bolotin, K. Sikes, Z. Jiang, M. Klima, G. Fudenberg, J. Hone, P. Kim, and H. Stormer, Solid State Commun. 146, 351 (2008).

[13] S. V. Morozov, K. S. Novoselov, M. I. Katsnelson, F. Schedin, D. C. Elias, J. A. Jaszczak, and A. K. Geim, Phys. Rev. Lett. 100, 016602 (2008).

[14] D. Svintsov, V. Vyurkov, S. Yurchenko, T. Otsuji, and V. Ryzhii, J. Appl. Phys. 111, 083715 (2012).

[15] F. M. D. Pellegrino, I. Torre, and M. Polini, Phys. Rev. B 96, 195401 (2017).

[16] R. Bistritzer and A.H. MacDonald, Phys. Rev. B 80, 085109 (2009).

[17] M. Müller, J. Schmalian, and L. Fritz, Phys. Rev. Lett. 103, 025301 (2009). 
[18] A. V. Andreev, S. A. Kivelson, and B. Spivak, Phys. Rev. Lett. 106, 256804 (2011).

[19] B. N. Narozhny, I. V. Gornyi, M. Titov, M. Schütt, and A. D. Mirlin, Phys. Rev. B 91, 035414 (2015).

[20] M. Müller and S. Sachdev, Phys. Rev. B 78, 115419 (2008).

[21] V. F. Gantmakher and I. B. Levinson, Sov. Phys. JETP 47, 133 (1978).

[22] J. C. W. Song, D. A. Abanin, and L. S. Levitov, Nano Lett. 13, 3631 (2013).

[23] D. A. Abanin, R. V. Gorbachev, K. S. Novoselov, A. K. Geim, and L. S. Levitov, Phys. Rev. Lett. 107, 096601 (2011).

[24] M. Titov, R. V. Gorbachev, B. N. Narozhny, T. Tudorovskiy, M. Schütt, P. M. Ostrovsky, I. V. Gornyi, A. D. Mirlin, M. I. Katsnelson, K. S. Novoselov, A. K. Geim, and L. A. Ponomarenko, Phys. Rev. Lett. 111, 166601 (2013).

[25] D. X. Nguyen, G. Wagner, and S. H. Simon, Phys. Rev. B 101, 035117 (2020).

[26] L. Fritz, J. Schmalian, M. Müller, and S. Sachdev, Phys. Rev. B 78, 085416 (2008).

[27] M. Müller, L. Fritz, and S. Sachdev, Phys. Rev. B 78, 115406 (2008).

[28] J. Lux and L. Fritz, Phys. Rev. B 86, 165446 (2012).

[29] J. Lux and L. Fritz, Phys. Rev. B 87, 075423 (2013).

[30] M. Zarenia, G. Vignale, T. B. Smith, and A. Principi, Phys. Rev. B 99, 161407 (2019).

[31] E. McCann and M. Koshino, Rep. Prog. Phys. 76, 056503 (2013).

[32] M. Koshino and T. Ando, Phys. Rev. B 73, 245403 (2006).

[33] E. McCann and V. I. Fal'ko, Phys. Rev. Lett. 96, 086805 (2006).

[34] Y. Nam, D.-K. Ki, D. Soler-Delgado, and A. F. Morpurgo, Science 362, 324 (2018).

[35] P. T. Dumitrescu, Phys. Rev. B 92, 121102(R) (2015).

[36] L. Spitzer and R. Härm, Phys. Rev. 89, 977 (1953).

[37] See Supplemental Material at http://link.aps.org/ supplemental/10.1103/PhysRevLett.124.026601 for a brief summary of the QBE formalism and the precise form of the collision integral used in the QBE. The Supplemental Material also contains a detailed derivation of the two-fluid model.

[38] $\kappa$ measures the heat current in the absence of electrical current.

[39] We also note that in Ref. [3], in three of four samples, $\sigma(\mu=0)$ does not vary more than about $10 \%$ over the full range of measured temperatures $10-100 \mathrm{~K}$.

[40] The dimensionless parameters for impurity scattering, scattering off the boundary and phonons respectively, are $\alpha_{\mathrm{imp}}=\left(8 \pi^{2} Z / N_{f} \epsilon_{r}\right)^{2} \beta n_{\mathrm{imp}} / m \alpha_{L}=(\sqrt{\beta} / \sqrt{m} L), \alpha_{\mathrm{ph}}=$ $\beta \tau_{\text {phonon }}^{-1}$. Here $L$ is the size of the sample, $Z$ is the charge of the impurities, $\epsilon_{r}$ is the relative permeability and $n_{\mathrm{imp}}$ is the impurity density.

[41] J. K. Viljas and T. T. Heikkilä, Phys. Rev. B 81, 245404 (2010).

[42] H. Ochoa, E. V. Castro, M. I. Katsnelson, and F. Guinea, Phys. Rev. B 83, 235416 (2011).

[43] S. S. Kubakaddi, Phys. Rev. B 79, 075417 (2009).

[44] R. Bistritzer and A. H. MacDonald, Phys. Rev. Lett. 102, 206410 (2009).

[45] H. Suzuura and T. Ando, Phys. Rev. B 65, 235412 (2002).

[46] H. Ochoa, E. V. Castro, M. I. Katsnelson, and F. Guinea, Phys. Rev. B 83, 235416 (2011).

[47] K. M. Borysenko, J. T. Mullen, X. Li, Y. G. Semenov, J. M. Zavada, M. B. Nardelli, and K. W. Kim, Phys. Rev. B 83, 161402(R) (2011).

[48] We note that the two-fluid model is equivalent to performing the Spitzer-Harm calculation with two basis functions.

[49] By considering the Lorenz force we can also include the magnetic field in this equation.

[50] J. Crossno, J. K. Shi, K. Wang, X. Liu, A. Harzheim, A. Lucas, S. Sachdev, P. Kim, T. Taniguchi, K. Watanabe, T. A. Ohki, and K. C. Fong, Science 351, 1058 (2016). 